\title{
A SIMPLE PROOF FOR THE BOUNDARY SCHWARZ LEMMA FOR PLURIHARMONIC MAPPINGS
}

\author{
Hidetaka Hamada \\ Kyushu Sangyo University, Faculty of Engineering \\ 3-1 Matsukadai, 2-Chome, Higashi-ku, Fukuoka 813-8503, Japan; h.hamada@ip.kyusan-u.ac.jp
}

\begin{abstract}
In this paper, we give a simple proof for the boundary Schwarz lemma for pluriharmonic mappings between Euclidean unit balls. We also give some generalization to $C^{1}$-mappings between domains with smooth boundaries.
\end{abstract}

\section{Introduction}

Let $B^{n}$ be the Euclidean unit ball in $\mathbf{C}^{n}$ and let $\mathbf{B}^{2 n}$ be the Euclidean unit ball in $\mathbf{R}^{2 n}$. Each $z=x+i y \in \mathbf{C}^{n}$ corresponds to $z^{\prime}=(x, y)^{T} \in \mathbf{R}^{2 n}$, where $T$ denotes the transpose of vectors and matrices. For $z \in \mathbf{C}^{n},\|z\|$ denotes the Euclidean norm on $\mathbf{C}^{n}$. For $x \in \mathbf{R}^{m},\|x\|$ denotes the Euclidean norm on $\mathbf{R}^{m}$. For each $z_{0}^{\prime} \in \partial \mathbf{B}^{2 n}$, the tangent space $T_{z_{0}^{\prime}}\left(\partial \mathbf{B}^{2 n}\right)$ is defined by

$$
T_{z_{0}^{\prime}}\left(\partial \mathbf{B}^{2 n}\right)=\left\{\beta \in \mathbf{R}^{2 n}: z_{0}^{\prime T} \beta=0\right\} .
$$

A $C^{2}$ mapping $f: B^{n} \rightarrow \mathbf{C}^{m}$ is said to be pluriharmonic if the restriction of each component $f_{j}$ to every complex line is harmonic.

Let $\Omega$ be a domain in $\mathbf{R}^{m}$. For a $C^{1}$ mapping $f: \Omega \rightarrow \mathbf{R}^{M}$, let $J_{f}(x)$ denote the $M \times m$ Jacobian matrix of $f$ at $x \in \Omega$.

In recent years, the Schwarz lemma at the boundary for holomorphic mappings has been studied by many authors $[1,2,3,5,6]$. More recently, the following boundary Schwarz lemma for pluriharmonic mappings between Euclidean unit balls was proved by Liu, Dai and Pan [4].

Theorem 1.1. Let $f: B^{n} \rightarrow B^{N}$ be a pluriharmonic mapping for $n, N \geq 1$. If $f$ is $C^{1+\alpha}$ at $z_{0} \in \partial B^{n}$ for some $\alpha \in(0,1)$ and $f\left(z_{0}\right)=w_{0} \in \partial B^{N}$, then we have

(I) $J_{f}\left(z_{0}^{\prime}\right) \beta \in T_{w_{0}^{\prime}}\left(\partial \mathbf{B}^{2 N}\right)$ for any $\beta \in T_{z_{0}^{\prime}}\left(\partial \mathbf{B}^{2 n}\right)$;

(II) There exists a positive $\lambda \in \mathbf{R}$ such that $J_{f}\left(z_{0}^{\prime}\right)^{T} w_{0}^{\prime}=\lambda z_{0}^{\prime}$,

where $z_{0}^{\prime}$ and $w_{0}^{\prime}$ are real versions of $z_{0}$ and $w_{0}$ respectively, and

$$
\lambda \geq \frac{1-\|f(0)\|}{2^{2 n-1}}>0 .
$$

For the proof, they used the Schwarz lemma for pluriharmonic mappings [4, Theorem 1.1], a technical lemma [4, Lemma 2.1] and the Harnack inequality for nonnegative harmonic functions on the Euclidean unit ball in $\mathbf{R}^{n}$ [4, Theorem 3.1]. In this paper, we will prove the following theorem by using the Harnack inequality for nonnegative harmonic functions on the unit disc $U$ in $\mathbf{C}$ and elementary arguments.

https://doi.org/10.5186/aasfm.2017.4245

2010 Mathematics Subject Classification: Primary 32U05, 30C80.

Key words: Boundary Schwarz lemma, pluriharmonic mapping.

H. Hamada was partially supported by JSPS KAKENHI Grant Number JP16K05217. 
We do not use the Schwarz lemma for pluriharmonic mappings [4, Theorem 1.1] and a technical lemma [4, Lemma 2.1].

The novelty of our theorem is as follows. We only need that $f$ is $C^{1}$ at $z_{0} \in \partial B^{n}$. Also, in (I), the assumption that $f$ is pluriharmonic is not needed. In (II), we give an improvement of the lower estimate for $\lambda$.

Theorem 1.2. Let $f: B^{n} \rightarrow B^{N}$ be a $C^{1}$ mapping for $n, N \geq 1$. Assume that $f$ is $C^{1}$ at $z_{0} \in \partial B^{n}$ and $f\left(z_{0}\right)=w_{0} \in \partial B^{N}$.

(I) Then we have $J_{f}\left(z_{0}^{\prime}\right) \beta \in T_{w_{0}^{\prime}}\left(\partial \mathbf{B}^{2 N}\right)$ for any $\beta \in T_{z_{0}^{\prime}}\left(\partial \mathbf{B}^{2 n}\right)$ and there exists a nonnegative $\lambda \in \mathbf{R}$ such that $J_{f}\left(z_{0}^{\prime}\right)^{T} w_{0}^{\prime}=\lambda z_{0}^{\prime}$;

(II) Moreover, if $f$ is pluriharmnic on $B^{n}$, then

$$
\lambda \geq \frac{1-\left(f(0)^{\prime}\right)^{T} w_{0}^{\prime}}{2} \geq \frac{1-\|f(0)\|}{2}>0 .
$$

We give an example of a real anaytic mapping $f$ such that $\lambda=0$ in (I) of the above theorem. So, we cannot conclude that $\lambda>0$ in (I) of the above theorem.

Example 1.3. Let $f(z)=\left(e^{-\left(x_{1}-1\right)^{2}}, 0, \ldots, 0\right)^{T}, f: B^{n} \rightarrow B^{N}$, where $z=$ $\left(x_{1}, \ldots, x_{n}\right)^{T}+i\left(y_{1}, \ldots, y_{n}\right)^{T}$. Let $z_{0}=(1, \ldots, 0)^{T} \in \partial B^{n}$. Then we have $w_{0}=$ $f\left(z_{0}\right)=(1, \ldots, 0)^{T} \in \partial B^{N}$ and $J_{f}\left(z_{0}^{\prime}\right)=O$. Therefore, $\lambda=0$.

We give a generalization of Theorem 1.2 (I) to $C^{1}$-mappings between domains with smooth boundaries. Let $\Omega$ be a domain in $\mathbf{R}^{n}$. $\Omega$ is said to have $C^{r}$ boundary $(r \geq 1)$, if there exist a neighbourhood $U$ of $\partial \Omega$ and a real valued $C^{r}$ function $\rho$ on $U$ such that $\Omega \cap U=\{x \in U: \rho(x)<0\}, \nabla \rho \neq 0$ on $\partial \Omega$, where

$$
\nabla \rho(x)=\left(\frac{\partial \rho}{\partial x_{1}}(x), \ldots, \frac{\partial \rho}{\partial x_{n}}(x)\right)^{T}, \quad \text { for } x=\left(x_{1}, \ldots, x_{n}\right)^{T} \in U .
$$

$\rho$ is called the defining function for $\Omega$. For each $x_{0} \in \partial \Omega$, the tangent space to $\partial \Omega$ at $x_{0}$ is defined as follows:

$$
T_{x_{0}}(\partial \Omega)=\left\{\beta \in \mathbf{R}^{n}: \nabla \rho\left(x_{0}\right)^{T} \beta=0\right\} .
$$

Proposition 1.4. Let $\Omega_{1} \subset \mathbf{R}^{m}$ be a domain with $C^{2}$-boundary and $\Omega_{2} \subset \mathbf{R}^{M}$ be a domain with $C^{1}$-boundary for $m, M \geq 1$. Let $\rho_{j}$ be the defining function for $\Omega_{j}$ for $j=1,2$, respectively. Let $f: \Omega_{1} \rightarrow \Omega_{2}$ be a $C^{1}$ mapping. Assume that $f$ is $C^{1}$ at $x_{0} \in \partial \Omega_{1}$ and $f\left(x_{0}\right)=y_{0} \in \partial \Omega_{2}$. Then we have $J_{f}\left(x_{0}\right) \beta \in T_{y_{0}}\left(\partial \Omega_{2}\right)$ for any $\beta \in T_{x_{0}}\left(\partial \Omega_{1}\right)$ and there exists a nonnegative $\lambda \in \mathbf{R}$ such that $J_{f}\left(x_{0}\right)^{T} \nabla \rho_{2}\left(y_{0}\right)=$ $\lambda \nabla \rho_{1}\left(x_{0}\right)$.

\section{Proof of Theorem 1.2}

Proof of (I). Let $\beta \in T_{z_{0}^{\prime}}\left(\partial \mathbf{B}^{2 n}\right)$ be fixed. We may assume that $\|\beta\|=1$. Let $\gamma(t)=\left(1-t^{2}\right) z_{0}^{\prime}+t \beta$. Then $\gamma(t) \in \mathbf{B}^{2 n}$ for $t \in(-1,1) \backslash\{0\}, \gamma(0)=z_{0}^{\prime}$ and $\left.\frac{d}{d t} \gamma(t)\right|_{t=0}=\beta$. Therefore the real valued function $\left(f(\gamma(t))^{\prime}\right)^{T} w_{0}^{\prime}$ attain its local maximum at $t=0$. Since this function is $C^{1}$ on $(-1,1)$, we have

$$
\left.\frac{d}{d t}\left(f(\gamma(t))^{\prime}\right)^{T} w_{0}^{\prime}\right|_{t=0}=\left(J_{f}\left(z_{0}^{\prime}\right) \beta\right)^{T} w_{0}^{\prime}=0 .
$$

This implies that $J_{f}\left(z_{0}^{\prime}\right) \beta \in T_{w_{0}^{\prime}}\left(\partial \mathbf{B}^{2 N}\right)$. Next, assume that $J_{f}\left(z_{0}^{\prime}\right)^{T} w_{0}^{\prime}=\lambda z_{0}^{\prime}+\beta$ for some $\lambda \in \mathbf{R}$ and $\beta \in T_{z_{0}^{\prime}}\left(\partial \mathbf{B}^{2 n}\right)$. Then

$$
\|\beta\|^{2}=\left(\lambda z_{0}^{\prime}+\beta\right)^{T} \beta=\left(J_{f}\left(z_{0}^{\prime}\right)^{T} w_{0}^{\prime}\right)^{T} \beta=w_{0}^{T} J_{f}\left(z_{0}^{\prime}\right) \beta=0
$$


by the above argument. Therefore, we have $J_{f}\left(z_{0}^{\prime}\right)^{T} w_{0}^{\prime}=\lambda z_{0}^{\prime}$ for some $\lambda \in \mathbf{R}$. Let

$$
u(\zeta)=1-\left(f\left(\zeta z_{0}^{\prime}\right)^{\prime}\right)^{T} w_{0}^{\prime}, \quad \zeta \in U .
$$

Then $u(r) \geq u(1)$ for $r \in(0,1)$. Therefore, we have

$$
\lambda=\left(J_{f}\left(z_{0}^{\prime}\right) z_{0}^{\prime}\right)^{T} w_{0}^{\prime}=\lim _{r \rightarrow 1-0} \frac{u(r)-u(1)}{1-r} \geq 0 .
$$

Proof of (II). Assume that $f$ is pluriharmonic on $B^{n}$. Then the function $u$ defined in (2.1) is nonnegative and harmonic on the unit disc $U$ in $\mathbf{C}$. By Harnack's inequality on the unit disc, we have

$$
\frac{1-r}{1+r} u(0) \leq u(\zeta) \leq \frac{1+r}{1-r} u(0), \quad \text { for } r=|\zeta|<1 .
$$

Therefore,

$$
\frac{1}{1+r} u(0) \leq \frac{u(r)-u(1)}{1-r}, \quad \text { for } 0<r<1
$$

Letting $r \rightarrow 1-0$, we have

$$
\frac{1-\left(f(0)^{\prime}\right)^{T} w_{0}^{\prime}}{2} \leq\left(J_{f}\left(z_{0}^{\prime}\right) z_{0}^{\prime}\right)^{T} w_{0}^{\prime}=\lambda
$$

This completes the proof.

\section{Proof of Proposition 1.4}

Let $\beta \in T_{x_{0}}\left(\partial \Omega_{1}\right)$ be fixed. Let $\gamma(t)=x_{0}+\varepsilon t \beta-t^{2} \nabla \rho_{1}\left(x_{0}\right)$. Then $\gamma(0)=x_{0}$ and $\left.\frac{d}{d t} \gamma(t)\right|_{t=0}=\varepsilon \beta$. Since $\Omega_{1}$ has $C^{2}$-boundary, there exist $\varepsilon>0$ and $t_{0}>0$ such that $\gamma(t) \in \Omega_{1}$ for $t \in\left(-t_{0}, t_{0}\right) \backslash\{0\}$. Therefore the real valued function $\rho_{2}(f(\gamma(t)))$ attain its local maximum at $t=0$. Since this function is $C^{1}$ near $t=0$, we have

$$
\left.\frac{d}{d t} \rho_{2}(f(\gamma(t)))\right|_{t=0}=\left(\nabla \rho_{2}\left(y_{0}\right)\right)^{T} J_{f}\left(x_{0}\right) \varepsilon \beta=0
$$

This implies that $J_{f}\left(x_{0}\right) \beta \in T_{y_{0}}\left(\partial \Omega_{2}\right)$. Next, assume that $J_{f}\left(x_{0}\right)^{T} \nabla \rho_{2}\left(y_{0}\right)=\lambda \nabla \rho_{1}\left(x_{0}\right)$ $+\beta$ for some $\lambda \in \mathbf{R}$ and $\beta \in T_{x_{0}}\left(\partial \Omega_{1}\right)$. Then

$$
\|\beta\|^{2}=\left(\lambda \nabla \rho_{1}\left(x_{0}\right)+\beta\right)^{T} \beta=\left(J_{f}\left(x_{0}\right)^{T} \nabla \rho_{2}\left(y_{0}\right)\right)^{T} \beta=\nabla \rho_{2}\left(y_{0}\right)^{T} J_{f}\left(x_{0}\right) \beta=0
$$

by the above argument. Therefore, we have $J_{f}\left(x_{0}\right)^{T} \nabla \rho_{2}\left(y_{0}\right)=\lambda \nabla \rho_{1}\left(x_{0}\right)$ for some $\lambda \in \mathbf{R}$. Since $x_{0}-t \nabla \rho_{1}\left(x_{0}\right) \in \Omega_{1}$ for sufficiently small $t>0$, we have

$$
\begin{aligned}
\lambda\left\|\nabla \rho_{1}\left(x_{0}\right)\right\|^{2} & =\left(\nabla \rho_{2}\left(y_{0}\right)\right)^{T} J_{f}\left(x_{0}\right) \nabla \rho_{1}\left(x_{0}\right) \\
& =-\left.\frac{d}{d t} \rho_{2}\left(f\left(x_{0}-t \nabla \rho_{1}\left(x_{0}\right)\right)\right)\right|_{t=0} \\
& =\lim _{t \rightarrow+0} \frac{\rho_{2}\left(f\left(x_{0}\right)\right)-\rho_{2}\left(f\left(x_{0}-t \nabla \rho_{1}\left(x_{0}\right)\right)\right)}{t} \\
& \geq 0 .
\end{aligned}
$$

Thus, $\lambda \geq 0$. This completes the proof. 


\section{References}

[1] Krantz, S. G.: The Schwarz lemma at the boundary. - Complex Var. Elliptic Equ. 56, 2011, $455-468$.

[2] LiU, T., and X. TANG: A new boundary rigidity theorem for holomorphic self-mappings of the unit ball in $\mathbf{C}^{n}$. - Pure Appl. Math. Q. 11, 2015, 115-130.

[3] LiU, T., J. WANG, and X. TANG: Schwarz lemma at the boundary of the unit ball in $\mathbf{C}^{n}$ and its applications. - J. Geom. Anal. 25, 2015, 1890-1914.

[4] LiU, Y., S. DAI, and Y. PAN: Boundary Schwarz lemma for pluriharmonic mappings between unit balls. - J. Math. Anal. Appl. 433, 2016, 487-495.

[5] TANG, X., and T. LiU: The Schwarz lemma at the boundary of the egg domain $B_{p_{1}, p_{2}}$ in $\mathbf{C}^{n}$. - Canad. Math. Bull. 58, 2015, 381-392.

[6] TAng, X., T. LiU, and J. Lu: Schwarz lemma at the boundary of the unit polydisk in $\mathbf{C}^{n}$. Sci. China Math. 58, 2015, 1639-1652.

Received 16 August $2016 \bullet$ Accepted 12 January 2017 